\title{
A comprehensive analysis of the polygalacturonase family of wheat (Triticum aestivum L.) shed light on important genes affecting pollen development and anther dehiscence
}

Jiali Ye

Northwest Agriculture and Forestry University

Xuetong Yang

Northwest Agriculture and Forestry University

Wei Li

Northwest Agriculture and Forestry University

Qi Liu

Northwest Agriculture and Forestry University

Fuqiang Niu

Northwest Agriculture and Forestry University

Yanru Chen

Northwest Agriculture and Forestry University

Lingli Zhang

Northwest Agriculture and Forestry University

Xiyue Song ( $\sim$ songxiyue@126.com )

Northwest Agriculture and Forestry University https://orcid.org/0000-0003-1307-4300

Research article

Keywords: gene family genome-wide pectin pollen polygalacturonase male sterility wheat

Posted Date: September 5th, 2019

DOI: https://doi.org/10.21203/rs.2.13948/v1

License: (c) (1) This work is licensed under a Creative Commons Attribution 4.0 International License.

Read Full License 


\section{Abstract}

Background: Polygalacturonase (PG) belongs to a large family of hydrolases that undertake many important functions in cell separation during plant growth and development by degrading pectin. The specific expression of PG genes in pollen may have great significance for plant male sterile research and hybrid wheat breeding. However, it has not been reported in wheat ( Triticum aestivum L.). Results: Therefore, we systematically studied the PG gene family using the latest published wheat reference genomic information. A total of 113 PGs were identified and renamed as TaPG01 - 113 based on their position on the chromosome. They were unequally distributed on 21 chromosomes and were classified into six categories of A-F. Analysis of gene structures and conserved motifs revealed that the TaPGs of Class $C$ and $D$ had relatively short gene sequences and a small number of introns, and Class $E$ TaPGs were the least conserved and all members did not have III conserved domain. Segmental duplication has been shown to be one of the major drivers of the expansion of the wheat PG gene family. The cis-element predictions indicate that wheat PGs had a wide range of functions, including response to light, hypothermia, anaerobic and hormonal stimulation, and also involved in meristematic tissue expression. In addition, twelve spike-specific expressions of TaPGs were screened using RNA-seq data, and finally three important genes were identified by expression analysis in the sterile and fertile anthers of thermosensitive male sterile wheat. TaPG93 was involved in the pollen development and elongation of pollen tubes, and TaPG87 and TaPG95 played important roles in the separation of pollen grains and the cracking of anthers dehiscence. Conclusions: This study, we performed a thorough analysis of the wheat PG gene family and finally obtained three TaPGs that affect wheat fertility. This will lay a solid foundation for the function exploration of wheat $P G$ gene family and provide new enlightenment for the fertility conversion mechanism of male sterile wheat.

\section{Background}

Polygalacturonase (PG, Ec, 3.2.1.15) is a cell wall binding protein that catalyzes the cleavage of a-(1,4)polygalacturonic acid in pectin. Among numerous enzymes that degrade pectin, PG belongs to the largest family of hydrolases. In 1956, PG was first studied and confirmed to be closely related to fruit softening. Subsequent studies have shown that the main functions of this enzyme is to degrade polygalacturonic acid in the cell wall of fruits into galactose and aldehyde acid, which disintegrates the cell wall structure and leads to fruit softening $[1,2]$. Fabi et al found that the overexpression of $c p-P G 1$ in papaya can promote the softening of pulp [3]. However, with the application of molecular biology technology in PG research, people have a deeper understanding of it. Roongsattham et al identified 14 PG genes during the maturation and shedding of oil palm fruit, all of which were expressed in the detachment of the fruit base at ripening [4]. A PG gene RDPG1 in Brassica napus was expressed mainly in the dehiscence zone of fruit pods and anthers, and also in the detachment zone of floral organs and the nodes of stems and pedicels during the growth of pollen tubes [5]. Arabidopsis thaliana PG gene QRT3 participates in the separation of tetrad microspores during the pollen development process by secreting and degrading pollen mother cell walls around the tetraploid microspores [6]. Bergey [7] and Orozco-Cardenas [8] suggested that injury 
induced the expression of PG genes, which produced plant-derived endogenous elicitors, and further activated defense genes PPO and pis in mesophyll cells. Those proved that members of the PG gene family can be expressed in different tissues and different stages of plant development, in addition to its role in fruit ripening, PGs are also associated with leaf and flower shedding, pod cracking, pollen maturation, pathogen defense, and plant host interaction $[9,10]$. Therefore, other functions of PGs on non-fruit plants are also worthy of further study, although its role in affecting fruit ripening has always been the focus of attention.

PGs can be classified into endo-polygalacturonase (endo-PG, Ec, 3.2.1.15), exo-polygalacturonase (exoPG) and rhamno-galactosidase (oligo-PG) depending on the site of the substrate [11]. According to the characteristic of amino acid sequences, Hadfield et al. classify PGs into three classes of A-C: the differentiation unit A consists of genes expressed in non-pollen tissues, which encode PG genes that lack of a pre-sequence proteins; the differentiation unit B consists of all PG genes encoding a pre-sequence, and the differentiation unit $C$ consists of $P G$ genes expressed in pollen, encoding exo-PGs [12]. Although this classification was determined by the PGs sequence, it also reflected the function differences of PGs, and different subclasses of PG have unique biological functions. However, Park et al. elaborated the division of $P G$ gene family members from algae to terrestrial plants through cluster analysis. 225 PG members from eight species were clustered into six branches from A to F. Class A and B contained PG genes from terrestrial plants, and class $E$ contained $P G$ genes from algae to flowering plants, while $C, D, F$ contained only the $P G$ genes of flowering plants. PGs of Class $A$ and Class $B$ are endo-PG, Class $C$ and Class D PGs are exdo-PG, Class E PGs are oligo-PG, while members of Class $F$ cannot be classified into any of properties of PG [11]. The A to F six class PG genes in Arabidopsis differ in expression patterns, with some class members tending to be expressed in vegetative tissues, while others tend to be expressed in reproductive organs [13]. Therefore, it is more detailed and convenient to screen functions related genes according to cluster classification rules, which is suitable for PG gene family analysis of most crops.

Based on the clustering classification rules, systematically analysis of the PG gene family have been carried out on Arabidopsis and rice [13], soybean [14], apple [15], poplar [16] and other crops, which provide great convenience for function mining of plant PG gene family. However, no analysis of the PG gene family has been reported in wheat. Wheat is a heterologous hexaploid organism with a total of 42 chromosomes, and the research progress was limited due to its large but incomplete genomic information. However, the completion of wheat genome sequencing and sequence release make it possible to explore and study important functional genes of wheat at the genome-wide level [17]. Since the pollen-specific PG genes in tobacco [18] and cotton [19] and Arabidopsis [20] have been studied, the study of wheat pollen-specific genes is well-founded and will provide a new perspective for wheat male sterile research.

KTM3315A is a thermo-sensitive male sterile wheat material that with excellent traits and great potential for hybrid production, which our team has always attached great importance to research. In the previous study, we found that KTM3315A showed male sterile under normal autumn sowing conditions in 
Shaanxi, China, but it showed fertility can be self-crossed during spring sowing in the same area. By microscopic observation, the binucleate stage was identified as a critical period of fertility conversion, from which the microspore mother cells of the sterile pollens exhibited abnormal shapes such as shrinkage and irregularity [21]. Transcriptome analysis proved that such abortive morphology was closely related to the expression of pectinase [21]. Obviously, it is necessary to study the pollen-specific PG genes that involved in pollen development mechanism through excellent male sterile materials, which can help us to cultivate more stable male sterile materials and accelerate the wheat hybridization breeding process.

In this study, a total of 113 members of wheat PG gene family were identified, and a systematic and comprehensive overview was performed through the analysis of its evolutionary relationships, gene structures, conserved domains, cis-elements and expression patterns. Based on the expression patterns in sterile and fertile anthers, we screened out three TaPGs related to pollen development and anther dehiscence. This is both pioneering and a meaningful study in wheat.

\section{Results}

\section{Identification and annotation of wheat PG gene family}

The Hidden Markov Model (HMM) file of Glycoside hydrolase family 28 (GH28, EC 3.2.1.) and the amino acid sequences of PGs of Arabidopsis were aligned with the whole amino acid sequences of wheat, and a total of 113 wheat PGs were finally obtained after strict screening and identification (Additional file 1: Table S1). These 113 wheat PGs were unevenly distributed on 21 chromosomes, and each chromosome had at least one PG. However, there were five PG genes distributed on the A, B and D of chromosomes 2 and 5 respectively, as well as chromosomes of 1D, 4A, and 4B. (Additional file 2: Figure S1). According to their chromosomal location, these 113 wheat PG genes were renamed as TaPG01-TaPG113. The 113 TaPGs have a CDS length of 762-1617 bp, and an exon number of 2-10. The molecular weight of wheat PG proteins ranged from 26.69-57.99 KDa, with a theoretical isoelectric point of 4.8-10.45, and encoding 253-538 amino acids (Table 1). Accurate identification and reasonable naming are essential for further research in this gene family.

\section{Evolutionary analysis of wheat PG gene family}

To understand the evolution pattern of wheat PG, a total of 238 PG proteins from wheat, Arabidopsis and rice were used to construct a phylogenetic tree (Fig. 1). The results showed that 238 PG proteins of these three species were clustered into six branches (A-F). Among these six classes, class A had 12 TaPGs, and class $B$ to class $F$ had $16,13,28,30$, and 14 TaPGs in sequence. In addition, there were 39 pairs of paralogous genes in 113 TaPGs, of which A class has four pairs of paralogous genes, class $\mathrm{B}, \mathrm{C}$ and $\mathrm{F}$ all had five pairs, while class $D$ and $E$ has nine and eleven pairs of paralogous genes respectively. However, there was only one pair of PG orthologous of rice and wheat (LOC_Os01g33300/TaPG44) in the D class. Therefore, it can be speculated that the expansion of the wheat PG family mainly occured after species differentiation from ancestor. 


\section{The duplications of wheat PGs}

In order to find out the expansion dynamics and evolution direction of the wheat PG gene family, we investigated its gene duplication events. The duplications of the wheat genome indicated that there were eight pairs of tandem duplications and 87 pairs of segmental duplications between TaPGs. The KA/KS values of these eight pairs of tandem duplication TaPGs were all less than 1, indicating that they were in the purify selection (Table 2). 93 TaPGs involved in segmental duplications, accounting for $82.30 \%$ (93/113) of all TaPGs and it is $34.58 \%$ higher than the probability of segmental duplications of whole wheat genome (number of all genes: 120744, number of segmental duplication genes: 57613 , percentage: 47.72\%) (Fig. 2, Additional file 3: Table S2). This is a good evidence that the segmental duplications promote the expansion of wheat PG gene family.

\section{Gene structure and conserved motif analysis of wheat PGs}

The alignment of amino acid sequences of 113 wheat PGs indicated that most of them contained four

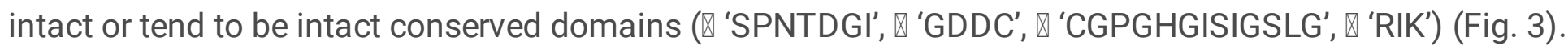
However, some PGs lack a certain domain, for example, in the B class, the amino acid sequences of the conserved domains of TaPG07 and TaPG81 were missing and incomplete. The most obvious deletions were shown in class E PGs, just as in the case of apple [15], the III conserved domains of all members of wheat class E PGs were missing. In addition, some of the conserved domains of PGs were complete, but individual amino acids were mutated. Most of the PGs in class $E$ mutated the serine $(S)$ to alanine $(A)$ or threonine $(T)$ in the conserved motif 'SPNTDGIH'. The domain IV was the most conserved region, except for the deletion in TaPG07 and the mutation of class E PGs, which completely conserved in the remaining TaPGs. In summary, the PGs in E class were the most variable members of the family.

MEME tool was used to search for the conserved motifs of 113 wheat PGs, and ten conserved motifs were obtained (Fig. 4, Additional file 4: Figure S2). No PG had all 10 motifs. Wheat PGs of class A and class $C$ had the same five motifs, while all class D wheat PG members had the same nine motifs except for TaPG92. It was found that motif 1 and motif 3 both contained conserved domain I 'SPNTDGI' and II 'GDDC' of PGs at the same time, and motif 1 also contained the portion of the conserved domain III 'CGPGHGISIGSLG', while motif 2 and motif 6 contained a conserved domain IV 'RIK'. In addition to the above four conserved motifs, motif 5 and motif 9 were the most conservative because they existed in almost all PGs including group E PGs. The structure of TaPGs showed that most of the PG genes had 1-9 introns, among which classes of $C$ and $D$ PGs belonging to exo-PG had relatively shorter gene sequences and fewer introns, and E class PGs belonging to oligo-PG generally had longer intron sequences, while class F PGs generally had more intron numbers (7-9). From the analysis of motifs and gene structures, not only the structural characteristics of each class of PGs can be obtained, but also provided a basis for classification of PG functions.

\section{GO annotation and cis-elements prediction of wheat PGs}


It is a very effective means to obtain corresponding gene functions by searching for annotation information in kind of databases and predicting upstream regulatory factors. We obtained the molecular function, biological process and cellular component of 113 TaPGs by searching for GO (Gene ontology) annotations. The GO annotations showed that most of the 113 TaPGs (68) were active in extracellular region, and all $113 \mathrm{TaPGs}$ exerted the hydrolysis and catalytic activity and involved in the primary metabolic and organic substance metabolic process (Fig. 5, Additional file 5: Table S3). Cis-acting elements of TaPGs were predicted, and ten of them were analyzed here. They included cis-elements responded for the anaerobic induction (ARE), light (G-box), low-temperature (LTR), also included ciselements regulated by hormones and transcription factors, such as cis-acting elements involved in the abscisic acid (ABRE), gibberellin (P-Box), auxin (TGA-element), methyl jasmonate (CGTCA-motif, TGACGmotif), and MYB binding site involved in drought-inducibility (MBS), as well as cis-acting regulatory element related to meristem expression(CAT-box). As can be seen from Figure 6, most TaPGs (85) may respond to light, $38 \mathrm{TaPGs}$ respond to low temperature, $83 \mathrm{TaPGs}$ respond to abscisic acid, $20 \mathrm{TaPGs}$ respond to gibberellin, and $32 \mathrm{TaPGs}$ participate in meristem expression.

\section{Analysis of the expression patterns of TaPGs}

To predict the relevant functions based on the expression specificity of PG, a heatmap were generated using RNA-seq data from Chinese spring wheat in 15 different developmental stages and tissues (Fig. 7). Obviously, 21, 7, 11 TaPGs were significantly up-regulated in the anthesis spikes (spike, reproductive, anthesis), spikes wrapped in flag leaves (spike, reproductive, flag leaf stage), and grains two days post anthesis (grain, reproductive, $2 \mathrm{dpa}$ ). These 21 up-regulated TaPGs in the anthesis spikes belonged to the D class, except for three from the B class. The 11 up-regulated TaPGs in spikes of the flag leaf stage were derived from the B, $\mathrm{C}$, and $\mathrm{D}$ classes, and the up-regulated TaPGs of the grain belonged to the $\mathrm{F}$ class. The results of these preference expressions strongly demonstrated that TaPGs of different classifications have a clear division of labor in different tissues. In order to explore TaPGS that play an important role in wheat pollen development, we focused on twelve completely spike-specific expressed TaPGs (TaPG09, $14,18,28,40,41,49,50,54,87,93,95)$.

\section{Pollen-specific TaPGs identified}

Real-time quantitative PCR (qRT-PCR) was performed on eight of spike-specific expressed TaPGs by sterile and fertile anthers of thermo-sensitive male sterile wheat KTM3315A to understand their expression modes in anthers. The results showed that three TaPGs (TaPG14, 40,41) that specifically expressed in the spikes of the flag leaf stage showed a decreasing trend with the development of anthers, while the anthesis spike-specific TaPGs (TaPG18, 28, 87, 93, 95) were up-regulated during the late anther development (Fig. 8). Just as confirmed by RT-PCR (Additional file 6: Figure S3), these differentially expressed data during anther development supported the fact that these spike-specific TaPGs were actually expressed in anthers. Although TaPG14, TaPG40 and TaPG41 were up-regulated at the uninucleate stage, their role in fertility conversion could be ruled out for they were not significantly differentially expressed between sterile and fertile anthers. TaPG18, TaPG28, TaPG87, TaPG93, and 
TaPG95 all had the highest expression levels at the trinucleate or binucleate stage in fertile anthers and showed significant differences in expression level with sterile anthers. However, the expression level of TaPG87 was significantly down-regulated in fertile anthers compared with sterile anthers, while the other four TaPGs were opposite. Their non-uniform expression patterns suggested that they had different roles in the development of anthers, and their functions were not redundant even if TaPG87 and TaPG95 all belong to class $B$ in this gene family.

By performing an alignment analysis on the above five differentially expressed $T a P G s$, we found that their protein sequences were highly similar to four Arabidopsis proteins (AT4G18180, PGA4, ADPG2, ADPG1), respectively. Like TaPG18 (401aa), the similarity between TaPG28 (401aa) and AT4G18180 (422a) was also bit-score 364.8, the bit-score of TaPG93 (294aa) and PGA4 (431aa, AT1G02790) was 263, TaPG87 (402aa) and ADPG2 (AT2G41850, 433aa) was 399.4, and TaPG95 (420aa) and ADPG1 (AT3G57510, 431aa) was 401. AT4G18180 belonged to the pectin lyase-like superfamily protein and its function was described as polygalacturonase activity, but its role in anthers has not been determined. However, PGA4 was identified as exo-polygalacturonase and played a role in the depolymerization of pectin during pollen development, pollen grain germination, and pollen tube growth. Its interaction protein included five pectin lyase-like superfamily proteins, two beta-D-xylosidase, two alpha-L-

arabinofuranosidase, and a glycosyl hydrolase 9A1 involved in cellulose biosynthesis. ADPG2 was identified as a polygalacturonase involved in cell separation in the final stages of pod shatter, in anther dehiscence and in floral organ abscission, and part of its interaction proteins have the function of organ shedding. ADPG1 was a polygalacturonase protein involved in silique and anther dehiscence, and most of its interaction proteins belonged to pectin lyase-like superfamily protein (Additional file 7: Table S4). Therefore, by aligning with the more well-studied model plant Arabidopsis to obtain sequence-similar Arabidopsis PG genes and predicting functions of the corresponding TaPGs, three TaPGs worth studying have broken into our field of vision. TaPG93 was significantly up-regulated during the binucleate stage and was supposed to be involved in pollen development and pollen tube growth, while TaPG87 and TaPG95, which were up-regulated in the trinucleate stage, played an essential role in pollen separation and anther dehiscence.

\section{Discussion}

Polygalacturonase, a glucosyl hydrolase family 28 , also known as pectic enzyme or pectin depolymerase. It is a gene family that is closely related to plant development, and playing roles by expressing them in different time and space, or by encoding different hydrolysis patterns or binding different substrates. In recent years, the development of genome sequencing has made it possible to systematically study the evolution, localization, structural and expression of plant PG genes. 66 PG genes were identified in Arabidopsis [12], 46 PG genes were identified in rice [13], 62 and 53 were identified in watermelon and cucumber [22], and 75, 85, 112, and 99 in Populus, apple, soybean, and Brassica rapa, respectively [14-16, 23]. In this study, we identified 113 wheat $P G$ genes and demonstrated that $P G$ gene family plays a complex role in higher plant wheat. The existence of genome-wide repeats and other repetitive events have led to the generation of many repetitive genes in the PG gene family during evolution [13]. Function 
redundancy and function differentiation may occur among numerous PG members due to the high degree of similarity of sequences [24]. From the duplication analysis of TaPGs, it can be seen there was higher synteny probability occurred between ABD sub-genomic in the same chromosome groups of wheat than that between different chromosome groups. There were eight pairs of tandem duplications and 87 pairs of segmental duplications in wheat PG gene family, and the proportion of segmental duplications of TaPGs was much higher than that of whole genomes. Therefore, we speculated that the main driving force for the expansion of wheat PG gene family was genome replication and segmental duplications.

Typical PGs have four conserved domains in plants and fungi: the amino acid sequences of domain I and domain II are 'SPNTDGI' and 'GDDC', respectively, and the three aspartic acid (D) of them may be components of the catalytic site [25]; the sequence of domain III is 'CGPGHGISIGSLG', in which the histidine residue $(\mathrm{H})$ is considered to be involved in the catalytic reaction [26]; the sequence of domain IV is 'RIK' with a positive charge, which may be involved in ionic interaction with the carboxy terminus of the substrate [27]. In general, a gene encoding a protein having the above four conserved domains is identified to belong PG gene family, but it is worth noting that the conservation of domain III is relatively poor [12]. This is consistent with our research. All TaPGs in class E did not have a conserved domain III, and only five of them kept completely conserved for remaining three domains, while others undergone at least one amino acid variation. Compared to other classes of PGs, class E PGs are widely present in algae to flowering plants, so they have experienced more natural selection pressures and more variation during the long evolutionary process.

PG acts as a downstream enzyme of the metabolic pathway, its expression and activity are regulated by other genes, proteins, plant hormones. In Arabidopsis, transcription factors BHLH, RPK2 AtDOF4.7 and AtC3H14 have been identified to regulate PG gene expression [20, 28-30]. In our research, we found that MYB was also involved in the regulation of PG. For the regulation of PGs by hormones, it has been studied in many species. Sun et al. inhibited the expression of SINCED1, a key enzyme in the synthesis of abscisic acid ( $A B A)$ in tomato, resulting in down-regulation of tomato PG gene [31]. In the transcriptome analysis of the ripening and shedding areas of oil palm fruit, it was shown that the expression abundance of EgPG4 increased by 700-5000 times in the base of the fruit when treated with ethylene [4]. Ziosi et al. applied MeJA to nectarine fruits at different developmental stages, which down-regulated the expression of fruit ripening genes including PGs, thereby delaying fruit ripening [32]. In Arabidopsis, When treated with jasmonate (JA), the expression levels of $A D P G 1, A D P G 2$, and QRT2 in the anthers were increased by about 10 times [20]. Although the hormones that regulate fruit ripening and shedding ultimately work by positively regulating PGs, the hormones that regulate PGs obtained by cis-element analysis in this study should behave as they were in Arabidopsis, which affected anther development and cracking.

In angiosperms, the main component of pollen inner wall is pectin, and the pollen tube wall is an extension of the inner wall of pollen[33]. Castro et al. used the monoclonal antibody LM5 immunolocalization technique revealed the presence of pectin in the pollen tube and pollen grains of olives, and found that the most abundant pectin was distributed in the three germinal holes [34, 35]. Along with the presence of pectin, many pectin degrading enzymes have been found in plant anthers, 
including pectin lyase, polygalacturonase, polymethylgalacturonase, pectin methylesterase [36-39]. As early as the 1990s, PG was found to be widespread in the late stages of plant anther development $[18,36$, $40,41]$, and the exploration of their action mechanism has been very frequent in recent years. BcMF2 was specifically expressed in the tapetum and pollen grains in the late tetrad, however, in the inhibited mutants bcmf2, the inner wall of the mature pollen grains was in a disordered state, the pollen tube failed to germinate normally, and the fertility of the plant was reduced [42]. BcMF9 was also expressed in the tapetum and microspores of late stage of pollen development, which affected the development of pollen and pollen tubes by affecting the formation of inner and outer walls [43]. In addition, in Chinese cabbage, BCMF6, BCMF16, and BcMF17 have also been identified as PG genes that play an important role in pollen development [44-46]. In Arabidopsis, three endo-PGs, including ADPG1 and ADPG2 and QRT2, were critical for pollen grains separation and contributed to anther dehiscence, but the overexpressing mutants of $Q R T 2$ caused male sterility [20].

In this study, by sequence similarity search, the protein sequences of TaPG87 and TaPG95 were found to be highly similar to the ADPG2 and ADPG1, respectively. From the qRT-PCR results, we found that they all expressed in the late stage (trinucleate) of fertile anthers just as many of the pollen-specific PGs that have been studied above, so we speculate that they are responsible for the separation of pollen grains and the cracking of anthers, like $A D P G 1$ and $A D P G 2$ in Arabidopsis. Although the down-regulated expression of of TaPG87 in fertile anthers is not as expected, the result have been repeatedly verified by RT-PCR (Additional file 6: Figure S3) and qRT-PCR. This suggested that different expression levels of TaPGs may have different effects on the regulation of anther development, just as moderate QRT2 promotes anther development but overexpression can lead to male sterile. Therefore, the effect of precise regulation of TaPG87 on wheat fertility deserves further study. The interacting proteins of PGA4 were several proteases that hydrolyze substrates, including $\beta$-D-xylosidase, a-L-arabinofuranosidase, glycosyl hydrolase and pectin lyase-like superfamily proteins. These enzymes have been shown to play important roles such as degrade the callosic wall surrounding the microspores and were closely related to the male fertility of plants [47-51]. The binucleate stage is a critical period for fertility conversion of thermosensitive male sterile wheat KTM3315A, which coincides with the highest expression stage of TaPG93 in fertile anthers. Therefore, combined with its similar protein PGA4 and interaction proteins as well as typical expression pattern, we identified TaPG93 as a key gene affecting pollen development and pollen tube growth, and also a crucial gene in KTM3315A during the fertility conversion process.

Therefore, based on this experiment, further subcellular localization and transgene verification of TaPG93 will be of great significance and our next task..

\section{Conclusions}

PG is a pectin hydrolase closely related to the development of plants involved in complex functions. It is important to understand pollen-specific PGs in wheat, which is extremely valuable for exploring pollen development mechanisms and the utilization of male sterile and heterosis. To systematically study wheat PG gene family, we identified a total of 113 PGs that were unevenly distributed on 21 chromosomes using the information of newly published wheat reference genomic. Based on the physical location, they were 
renamed as TaPG01-TaPG113. According to the phylogenetic tree constructed with Arabidopsis and rice, 113 TaPGs were divided into six branches of A-G, of which class E PGs had the lowest conservation and no III conserved domains. Duplications analysis revealed that one of the main drivers of wheat PG gene family expansion was segmental duplication. The analysis of cis-elements revealed the response of TaPGs to light, anaerobic, low temperature and other environments, and it also provided a new basis for studying the regulation of wheat PG by hormones. Through expression analysis, twelve spike-specific TaPGs were identified. After further analysis, these spike-specific genes were actually expressed in anther and three of them were predicted as important roles in pollen development and anther dehiscence. This provided an important theoretical basis for cultivating excellent male sterile materials.

\section{Methods}

\section{Plant Materials}

In this experiment, we used a wheat material KTM3315A selected by our research team in 2001 at the Northwest A\&F University of Yangling, Shaanxi, China. KTM3315A is a thermo-sensitive male sterile wheat material that our research group has been focusing on because of its strong agronomic traits and stable fertility performance. It exhibits sterile in low temperature conditions and fertile under conditions of high temperature, so it can both achieve self-crossing and be an advantageous materials for heterosis utilization [21]. Each 10 pots of wheat KTM3315A were placed in two artificial climate incubators with parameters set to $14 \mathrm{~h}$ light (day) and $10 \mathrm{~h}$ dark (night), and the day/night temperatures were $17{ }^{\circ} \mathrm{C} / 15^{\circ} \mathrm{C}$ (sterile condition) and $22^{\circ} \mathrm{C} / 20^{\circ} \mathrm{C}$ (fertile condition), respectively. A total of 20 pots of KTM3315A were treated at different temperatures until grouting was completed. The roots, stems, leaves and anthers from both sterile and fertile plants in three stages (uninucleate, binucleate and trinucleate) were saved in the $-80{ }^{\circ} \mathrm{C}$ refrigerator, as well as freshly grouted grains of fertile plants.

\section{Identification of wheat PGs gene family}

The whole protein sequences of wheat and the HMM file of GH28 were downloaded from the Ensembl plant (http://plants.ensembl.org/Triticum_aestivum/Info/Index) [52] and Pfam databases (http://pfam.xfam.org/family/PF00295) [53], respectively. A wheat-specific HMM file was constructed using the results of the wheat protein sequences alignment with the GH28 HMM file with the e-value was less than 1e-20. Then, The newly constructed wheat-specific HMM file was used again to align with the wheat protein sequences, and the results with an e-value of less than 0.01 were identified as candidate wheat PGs. To avoid omissions, we aligned whole wheat protein sequences with PGs sequences of Arabidopsis that downloaded from phytozome [13]. The above two strategies obtained all candidate wheat PGs, and the final wheat PGs were identified after screening by CD-search (https://www.ncbi.nlm.nih.gov/Structure/bwrpsb/bwrpsb.cgi) [54], SMART (http://smart.embl.de/) [55] and pfam (http://pfam.xfam.org/) [53]. Their genetic location information were obtained from reference genome annotation. The amino acid length of PG proteins, molecular weight and isoelectric point were predicted by Expsy (https://www.expasy.org/structural_bioinformatics). 


\section{Constructing phylogenetic tree of wheat PGs, and analysis of gene duplications}

In order to sort out the evolutionary relationship of wheat PGs, the amino acid sequences of 66 Arabidopsis PGs, 59 rice PGs and 114 wheat PGs were performed for clustalw sequence alignment and phylogenetic tree construction by MAGE. The phylogenetic tree was constructed by using the NeighborJoining $(\mathrm{NJ})$ method with parameters were set as repeated with bootstrap 1000 times. Tandem duplications and segmental duplications of the wheat PG gene family were obtained by Mcscanx [56] and the rations of the nonsynonymous substitution rate to the synonymous substitution rate (KA/KS) of tandem duplication PGs were calculated by the KaKs_Calculator2.0 [57].

\section{Analysis of gene structure and conserved domain of wheat PGs}

From multiple sequence alignment analysis of conserved regions, we obtained four conserved domains of the PG gene family and visualized them using GeneDoc software. Using MEME, 10 conserved motifs with lengths of 6-50 were obtained. Combined with the wheat genome annotation information, the genetic structure and conserved motifs of wheat PGs were visualized by TB-tools [58].

\section{GO annotation, cis-element and function predictions of TaPGs}

To understand the molecular functions, biological Processes and cellular components of wheat PGs, the GO annotation of wheat PGs were obtained from ensembl plant. It is helpful to understand the upstream regulatory factors of PG by analyzing cis-elements. Therefore, the $1500 \mathrm{bp}$ sequences of upstream of the TaPGs promoter were used to predict the cis-elements of PGs via plantCARE (http://bioinformatics.psb.ugent.be/webtools/plantcare/html/) [59]. To predict the function of TaPGs, STRING was used to obtain Arabidopsis PGs that with similar sequences to wheat PGs, and their functions and interactions [60].

\section{Expression analysis of TaPGs}

In order to screen genes based on expression preference, RNA-seq data from 15 different tissues (root, stem, leaf, spike, grain) and developmental stages (seedling, vegetative, reproductive) of Chinese spring were used to analyze the expression profile of wheat PG genes [61]. To further verify whether spikespecific TaPGs are associated with anther development and fertility conversion, a relative quantitative analysis of sterile and fertile anthers was performed on qRT-PCR in three stages (uninucleate, binucleate, trinucleate). Total RNA was extracted using TRIGene reagent (GeneStar), and reverse transcription of CDNA and RT-PCR were performed by StarScript II First-strand cDNA Synthesis Kit-II (GeneStar) and 2×RealStar Green Fast Mixture (GeneStar), respectively. All technical procedures were referred to the manufacturer's instructions. We used the wheat Actin gene as an internal reference and calculated the relative expression level of each gene in sterile and fertile plants by $2^{\Delta \Delta C t}$ based on the $\mathrm{Ct}$ (cycle threshold) value obtained from the Applied Biosystems 7500 Real-Time PCR System.

\section{Abbreviations}


ABA: Abscisic acid; Ct: cycle threshold; dpa: days post anthesis; endo-PG: endo-polygalacturonase ; exoPG: exo-polygalacturonase; GH28: Glucosyl hydrolase family 28; GO: Gene ontology; HMM: Hidden Markov Model ; JA: jasmonate; Ka: nonsynonymous substitution rate; Ks: synonymous substitution rate; oligo-PG: rhamno-galactosidase; PG: Polygalacturonase

\section{Declarations}

\section{Ethics approval and consent to participate}

Not applicable.

\section{Consent for publication}

Not applicable.

\section{Availability of data and materials}

The datasets generated and analyzed during the current study are available from the corresponding author on reasonable request

\section{Competing interest}

The authors declar that they have no competing interests

\section{Funding}

the Program in Science and Technology of Yangling State Demonstration Zone of Agricultural High-tech Industries (2018NY-19)

\section{Authors' contributions}

XS and LZ designed the research. JY performed the experiments. JY, XY, WL and QL analyzed the data. JY wrote the manuscript with contributions from all the authors. XS critically revised the manuscript. All authors have read and approved the manuscript.

\section{Acknowledgements}

Not applicable.

\section{References}

1. Hadfield KA, Rose JKC, Yaver DS, Berka R, Bennett AB: Polygalacturonase Gene Expression in Ripe Melon Fruit Supports a Role for Polygalacturonase in Ripening-Associated Pectin Disassembly. Plant Physiology 1998, 117(2):363-373. 
2. Rose JKC, Hadfield KA, Labavitch JM, Bennett AB: Temporal Sequence of Cell Wall Disassembly in Rapidly Ripening Melon Fruit. Plant Physiology 1998, 117(2):345-361.

3. Fabi JP, Broetto SG, Silva SLGLD, Zhong S, Lajolo FM, Nascimento JROD: Analysis of papaya cell wall-related genes during fruit ripening indicates a central role of polygalacturonases during pulp softening. PLOS ONE 2014, 9(8).

4. Roongsattham P, Morcillo F, Jantasuriyarat C, Pizot M, Moussu S, Jayaweera D, Collin M, Gonzalezcarranza ZH, Amblard P, Tregear J: Temporal and spatial expression of polygalacturonase gene family members reveals divergent regulation during fleshy fruit ripening and abscission in the monocot species oil palm. BMC Plant Biology 2012, 12(1):150-150.

5. Sander L, Child R, Ulvskov P, Albrechtsen M, Borkhardt B: Analysis of a dehiscence zone endopolygalacturonase in oilseed rape (Brassica napus) and Arabidopsis thaliana: evidence for roles in cell separation in dehiscence and abscission zones, and in stylar tissues during pollen tube growth. Plant Molecular Biology 2001, 46(4):469.

6. Rhee SY, Osborne E, Poindexter P, Somerville C: Microspore Separation in the quartet 3 Mutants of Arabidopsis Is Impaired by a Defect in a Developmentally Regulated Polygalacturonase Required for Pollen Mother Cell Wall Degradation. Plant Physiology 2003, 133(3):1170-1180.

7. Bergey DR, Orozcocardenas ML, Moura DS, Ryan CA: A wound- and systemin-inducible polygalacturonase in tomato leaves. Proceedings of the National Academy of Sciences of the United States of America 1999, 96(4):1756-1760.

8. Orozcocardenas ML, Narvaezvasquez J, Ryan CA: Hydrogen Peroxide Acts as a Second Messenger for the Induction of Defense Genes in Tomato Plants in Response to Wounding, Systemin, and Methyl Jasmonate. The Plant Cell 2001, 13(1):179-191.

9. Mohnen D: Pectin structure and biosynthesis. Current Opinion in Plant Biology 2008, 11(3):266-277.

10. Ogawa M, Kay P, Wilson SM, Swain SM: ARABIDOPSIS DEHISCENCE ZONE POLYGALACTURONASE1 (ADPG1), ADPG2, and QUARTET2 are polygalacturonases required for cell separation during reproductive development in Arabidopsis. The Plant Cell 2009, 21(1):216-233.

11. Park K, Kwon S, Kim N: Intron loss mediated structural dynamics and functional differentiation of the polygalacturonase gene family in land plants. Genes \& Genomics 2010, 32(6):570-577.

12. Park KCPC, Kwon SJKJ, Kim PHKH, Bureau TB, Kim NSKS: Gene structure dynamics and divergence of the polygalacturonase gene family of plants and fungus. Genome 2008, 51(1):30-40.

13. Kim J, Shiu SH, Thoma S, Li W, Patterson SE: Patterns of expansion and expression divergence in the plant polygalacturonase gene family. Genome Biology 2006, 7(9).

14. Wang F, Sun X, Shi X, Zhai H, Tian C, Kong F, Liu B, Yuan X: A Global Analysis of the Polygalacturonase Gene Family in Soybean (Glycine max). PLOS ONE 2016, 11(9).

15. Chen H, Shao H, Fan S, Ma J, Zhang D, Han M: Identification and Phylogenetic Analysis of the POLYGALACTURONASE Gene Family in Apple. Horticultural Plant Journal 2016, 2(5):241-252.

16. Yang Z, Liu H, Wang X, Zeng Q: Molecular evolution and expression divergence of the Populus polygalacturonase supergene family shed light on the evolution of increasingly complex organs in 
plants. New Phytologist 2013, 197(4):1353-1365.

17. Appels R, Eversole K, Stein N, Feuillet C, Keller B, Rogers J, Pozniak CJ, Choulet F, Distelfeld A, Poland $\mathrm{J}$ et al: Shifting the limits in wheat research and breeding using a fully annotated reference genome. Science 2018, 361.

18. Tebbutt SJ, Rogers HJ, Lonsdale DM: Characterization of a tobacco gene encoding a pollen-specific polygalacturonase. Plant Molecular Biology 1994, 25(2):283-297.

19. John ME, Petersen MW: COTTON (GOSSYPIUM HIRSUTUM L.) POLLEN-SPECIFIC POLYGALACTURONASE MRNA : TISSUE AND TEMPORAL SPECIFICITY OF ITS PROMOTER IN TRANSGENIC TOBACCO. Plant Molecular Biology 1994, 26(6):1989-1993.

20. Ogawa M, Kay P, Wilson S, Swain SM: ARABIDOPSIS DEHISCENCE ZONE POLYGALACTURONASE1 (ADPG1), ADPG2, and QUARTET2 are Polygalacturonases required for cell separation during reproductive development in Arabidopsis. The Plant cell 2009, 21(1):216-233.

21. Ye J, Duan Y, Hu G, Geng X, Zhang G, Yan P, Liu Z, Zhang L, Song X: Identification of Candidate Genes and Biosynthesis Pathways Related to Fertility Conversion by Wheat KTM3315A Transcriptome Profiling. Frontiers in Plant Science 2017, 8(449).

22. Yu Y, Liang Y, Lv M, Wu J, Lu G, Cao J: Genome-wide identification and characterization of polygalacturonase genes in Cucumis sativus and Citrullus lanatus. Plant Physiology and Biochemistry 2014, 74:263-275.

23. Liang Y, Yu Y, Shen X, Dong H, Lyu M, Xu L, Ma Z, Liu T, Cao J: Dissecting the complex molecular evolution and expression of polygalacturonase gene family in Brassica rapa ssp. chinensis. Plant Molecular Biology 2015, 89(6):629-646.

24. Kim J, Patterson SE: Expression Divergence and Functional Redundancy of Polygalacturonases in Floral Organ Abscission. Plant Signaling \& Behavior 2006, 1(6):281-283.

25. Rexovabenkova L: Evidence for the role of carboxyl groups in activity of endopolygalacturonase of Aspergillus niger . Chemical modification by carbodiimide reagent. Collection of Czechoslovak Chemical Communications 1990, 55(5):1389-1395.

26. Rao MN, Kembhavi AA, Pant A: Implication of tryptophan and histidine in the active site of endopolygalacturonase from Aspergillus ustus: elucidation of the reaction mechanism. Biochimica et Biophysica Acta 1996, 1296(2):167-173.

27. Bussink HJD, Buxton FP, Visser J: Expression and sequence comparison of the Aspergillus niger and Aspergillus tubigensis genes encoding polygalacturonase II. Current Genetics 1991, 19(6):467-474.

28. Wei P, Tan F, Gao X, Zhang X, Wang G, Xu H, Li L, Chen J, Wang X: Overexpression of AtDOF4.7, an Arabidopsis DOF Family Transcription Factor, Induces Floral Organ Abscission Deficiency in Arabidopsis. Plant Physiology 2010, 153(3):1031-1045.

29. Kim WC, Kim JY, Ko J, Kang H, Kim JH, Han KH: AtC3H14, a plant-specific tandem CCCH zinc-finger protein, binds to its target mRNAs in a sequence-specific manner and affects cell elongation in Arabidopsis thaliana. Plant Journal 2014, 80(5):772-784. 
30. Mizuno S, Osakabe Y, Maruyama K, Ito T, Osakabe K, Sato T, Shinozaki K, Yamaguchishinozaki K: ReceptortAPlike protein kinase 2 (RPK 2) is a novel factor controlling anther development in Arabidopsis thaliana. Plant Journal 2007, 50(5):751-766.

31. Sun L, Sun Y, Zhang M, Wang L, Ren J, Cui M, Wang Y, Ji K, Li P, Li Q: Suppression of 9-cisEpoxycarotenoid Dioxygenase, Which Encodes a Key Enzyme in Abscisic Acid Biosynthesis, Alters Fruit Texture in Transgenic Tomato. Plant Physiology 2012, 158(1):283-298.

32. Ziosi V, Bonghi C, Bregoli AM, Trainotti L, Biondi S, Sutthiwal S, Kondo S, Costa G, Torrigiani P: Jasmonate-induced transcriptional changes suggest a negative interference with the ripening syndrome in peach fruit. Journal of Experimental Botany 2008, 59(3):563-573.

33. Taylor LP, Hepler PK: POLLEN GERMINATION AND TUBE GROWTH. Annual Review of Plant Biology 1997, 48(1):461-491.

34. Owen HA, Makaroff CA: Ultrastructure of microsporogenesis and microgametogenesis inArabidopsis thaliana (L.) Heynh. ecotype Wassilewskija (Brassicaceae). Protoplasma 1995, 185:7-21.

35. Castro AJ, Suarez C, Zienkiewicz K, Alche JDD, Zienkiewicz A, Rodriguezgarcia MI: Electrophoretic profiling and immunocytochemical detection of pectins and arabinogalactan proteins in olive pollen during germination and pollen tube growth. Annals of Botany 2013, 112(3):503-513.

36. Brown SM, Crouch ML: Characterization of a gene family abundantly expressed in Oenothera organensis pollen that shows sequence similarity to polygalacturonase. The Plant Cell 1990, 2(3):263-274.

37. Albani D, Altosaar I, Arnison PG, Fabijanski SF: A gene showing sequence similarity to pectin esterase is specifically expressed in developing pollen of Brassica napus. Sequences in its $5^{\prime}$ flanking region are conserved in other pollen-specific promoters. Plant Molecular Biology 1991, 16(4):501-513.

38. Sexton R, Campillo ED, Duncan D, Lewis LN: The purification of an anther cellulase ( $\beta$-1,4-glucan 4glucanohydrolase) from Lathyrus odoratus $L$. and its relationship to the similar enzyme found in abscission zones. Plant Science 1990, 67(2):169-176.

39. Neelam A, Sexton R: Cellulase (Endo $\beta-1,4$ Glucanase) and Cell Wall Breakdown during Anther Development in the Sweet Pea (Lathyrus odoratus L.): Isolation and Characterization of Partial cDNA Clones. Journal of Plant Physiology 1995, 146:622-628.

40. Allen RL, Lonsdale DM: Sequence analysis of three members of the maize polygalacturonase gene family expressed during pollen development. Plant Molecular Biology 1992, 20(2):343-345.

41. Robert LS, Allard S, Gerster JL, Cass L, Simmonds J: Isolation and characterization of a polygalacturonase gene highly expressed in Brassica napus pollen. Plant Molecular Biology 1993, 23(6):1273-1278.

42. Huang L, Cao J, Zhang A, Ye Y, Zhang Y, Liu T: The polygalacturonase gene BcMF2 from Brassica campestris is associated with intine development. Journal of Experimental Botany 2009, 60(1):301313.

43. Huang $L, Y e Y$, Zhang $Y$, Zhang A, Liu T, Cao J: BcMF9, a novel polygalacturonase gene, is required for both Brassica campestris intine and exine formation. Annals of Botany 2009, 104(7):1339-1351. 
44. Zhang Q, Huang L, Liu T, Yu X, Cao J: Functional analysis of a pollen-expressed polygalacturonase gene BcMF6 in Chinese cabbage (Brassica campestris L. ssp. chinensis Makino). Plant Cell Reports 2008, 27(7):1207-1215.

45. Zhang A, Qiu L, Huang L, Yu X, Lu G, Cao J: Isolation and Characterization of an Anther-Specific Polygalacturonase Gene, BcMF16, in Brassica campestris ssp. chinensis. Plant Molecular Biology Reporter 2012, 30(2):330-338.

46. Zhang A, Chen Q, Huang L, Qiu L, Cao J: Cloning and Expression of an Anther-Abundant Polygalacturonase Gene BcMF17 from Brassica Campestris ssp. Chinensis. Plant Molecular Biology Reporter 2011, 29(4):943-951.

47. Worrall D, Hird DL, Hodge R, Paul W, Draper J, Scott RG: Premature dissolution of the microsporocyte callose wall causes male sterility in transgenic tobacco. The Plant Cell 1992, 4(7):759-771.

48. Lalanne E, Honys D, Johnson A, Borner GHH, Lilley KS, Dupree P, Grossniklaus U, Twell D: SETH1 and SETH2, Two Components of the Glycosylphosphatidylinositol Anchor Biosynthetic Pathway, Are Required for Pollen Germination and Tube Growth in Arabidopsis. The Plant Cell 2004, 16(1):229240.

49. Coimbra S, Costa MRPFN, Jones B, Mendes MA, Pereira LG: Pollen grain development is compromised in Arabidopsis agp6 agp11 null mutants. Journal of Experimental Botany 2009, 60(11):3133-3142.

50. Hrub -б P, Honys D, Twell D, Capkov Fб V, Tup H- J: Expression of $\beta$-galactosidase and $\beta$-xylosidase genes during microspore and pollen development. Planta 2005, 220:931-940.

51. Nguyen TD, Moon S, Oo MM, Tayade R, Soh M, Song JT, Oh SA, Jung K, Park SK: Application of rice microspore-preferred promoters to manipulate early pollen development in Arabidopsis: a heterologous system. Sexual Plant Reproduction 2016, 29(4):291-300.

52. Kersey P, Allen JE, Allot A, Barba M, Boddu S, Bolt BJ, Carvalhosilva D, Christensen MB, Davis P, Grabmueller C: Ensembl Genomes 2018: an integrated omics infrastructure for non-vertebrate species. Nucleic Acids Research 2018, 46.

53. El-Gebali S, Mistry J, Bateman A, Eddy SR, Luciani A, Potter SC, Qureshi M, Richardson LJ, Salazar GA, Smart A et al: The Pfam protein families database in 2019. Nucleic Acids Research 2018, 47(D1):D427-D432.

54. Marchlerbauer A, Lu S, Anderson JB, Chitsaz F, Derbyshire MK, Deweesescott C, Fong JH, Geer LY, Geer RC, Gonzales NR: CDD: a Conserved Domain Database for the functional annotation of proteins. Nucleic Acids Research 2011, 39:225-229.

55. Letunic I, Bork P: 20 years of the SMART protein domain annotation resource. Nucleic Acids Research 2018, 46.

56. Wang Y, Tang H, Debarry JD, Tan X, Li J, Wang X, Lee T, Jin H, Marler BS, Guo H: MCScanX: a toolkit for detection and evolutionary analysis of gene synteny and collinearity. Nucleic Acids Research 2012, 40(7). 
57. Guo J, Jain R, Yang P, Fan R, Kwoh CK, Zheng J: Reliable and fast estimation of recombination rates by convergence diagnosis and parallel Markov Chain Monte Carlo. IEEE/ACM Transactions on Computational Biology and Bioinformatics 2014, 11(1):63-72.

58. Chen $\mathrm{C}$, Xia R, Chen H, He Y: TBtools, a Toolkit for Biologists integrating various HTS-data handling tools with a user-friendly interface. bioRxiv 2018:289660.

59. Lescot M, Dehais P, Thijs G, Marchal K, Moreau Y, De Peer YV, Rouze P, Rombauts S: PlantCARE, a database of plant cis-acting regulatory elements and a portal to tools for in silico analysis of promoter sequences. Nucleic Acids Research 2002, 30(1):325-327.

60. Von Mering C, Jensen LJ, Snel B, Hooper SD, Krupp M, Foglierini M, Jouffre N, Huynen MA, Bork P: STRING: known and predicted proteintAУprotein associations, integrated and transferred across organisms. Nucleic Acids Research 2004, 33:433-437.

61. Choulet F, Alberti A, Theil S, Glover N, Barbe V, Daron J, Pingault L, Sourdille P, Couloux A, Paux E: Structural and functional partitioning of bread wheat chromosome 3B. Science 2014, 345(6194):1249721-1249721.

\section{Tables}

Table 1. Annotation of 113 TaPGs. 


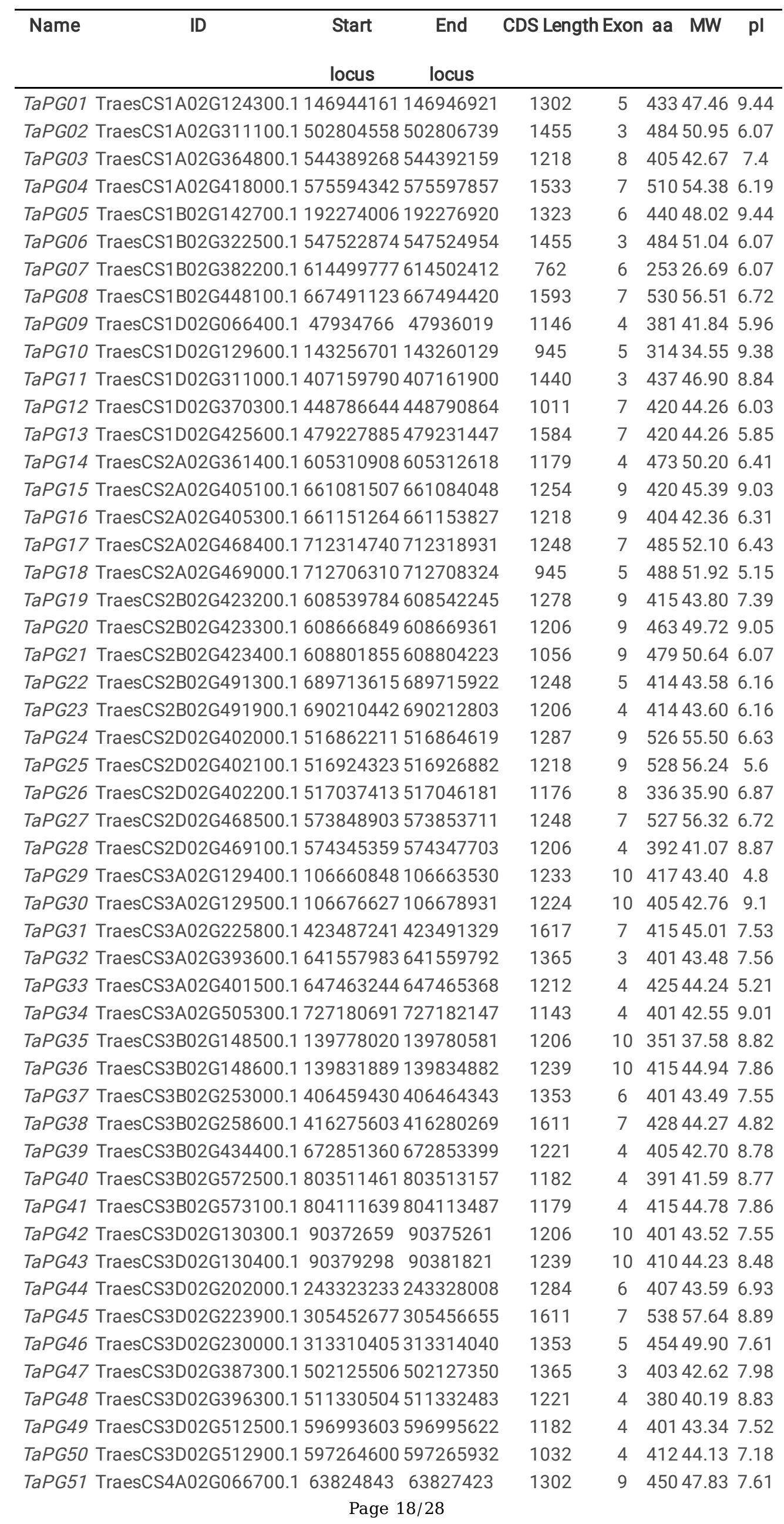


TaPG52 TraesCS4A02G168800.1423154931423158789 TaPG53 TraesCS4A02G229600.1538839203538840998 TaPG54 TraesCS4A02G229700.1538904932538906842 TaPG55 TraesCS4A02G358700.1631920845 631925008 TaPG56 TraesCS4B02G086400.186359231 86361142 TaPG57 TraesCS4B02G086500.1 8646504086466774 TaPG58 TraesCS4B02G144700.1195460952 195464972 TaPG59 TraesCS4B02G244300.1505543818 505546374 TaPG60 TraesCS4B02G344700.1638122183638125126 TaPG61 TraesCS4D02G146700.1136183828 136187858 TaPG62 TraesCS4D02G243600.1406231167406233698 TaPG63 TraesCS5A02G090800.1123708094123713351 TaPG64 TraesCS5A02G212900.1428148072 428151619 TaPG65 TraesCS5A02G264400.1476667366 476670027 TaPG66 TraesCS5A02G468900.1646057598 646059720 TaPG67 TraesCS5A02G513600.1677160384677163913 TaPG68 TraesCS5B02G097100.1 128215578128219271 TaPG69 TraesCS5B02G210900.1381717109 381720627 TaPG70 TraesCS5B02G263700.1448130267448132946 TaPG71 TraesCS5B02G481500.1 654365679654367442 TaPG72 TraesCS5B02G513100.1678335575678339587 TaPG73 TraesCS5D02G102900.1116170170116175261 TaPG74 TraesCS5D02G219200.1328436236328439556 TaPG75 TraesCS5D02G271900.1375309726375312492 TaPG76 TraesCS5D02G481800.1519544686519546837 TaPG77 TraesCS5D02G513600.1537671501537675675 TaPG78 TraesCS6A02G084500.153077600 53082399 TaPG79 TraesCS6A02G159000.1150980807150982536 TaPG80 TraesCS6A02G340000.1573405382 573408916 TaPG81 TraesCS6B02G118000.1112897663112903149 TaPG82 TraesCS6B02G192300.1226395703226397460 TaPG83 TraesCS6B02G371200.1 645351055645353904 TaPG84 TraesCS6D02G153500.1127379138127380712 TaPG85 TraesCS6D02G320600.1428641253428644718 TaPG86 TraesCS7A02G019400.1 79367587939018 TaPG87 TraesCS7A02G162500.1118462549 118464330 TaPG88 TraesCS7A02G270000.1275971899 275973610 TaPG89 TraesCS7A02G323800.1470999122 471003850 TaPG90 TraesCS7A02G330000.1482216406 482219390 TaPG91 TraesCS7A02G404700.1586691986586693761 TaPG92 TraesCS7A02G404800.1587364624587366468 TaPG93 TraesCS7A02G404900.1587518498587520054 TaPG94 TraesCS7A02G405000.1588738598588740487 TaPG95 TraesCS7B02G067800.173134816 73136777 TaPG96 TraesCS7B02G168200.1234912943234914373 TaPG97 TraesCS7B02G224700.1424565499 424569003 TaPG98 TraesCS7B02G230600.1433062559433065753 TaPG99 TraesCS7B02G304100.1544881057544882860 TaPG100 TraesCS7B02G304200.1544911423544913191 TaPG101 TraesCS7B02G304400.1544982183544983951 TaPG102 TraesCS7B02G304500.1545973515545975328 TaPG103TraesCS7D02G016300.1 $7171203 \quad 7173625$ TaPG104TraesCS7D02G164200.1113446046113448164
6 8.89

440643.057 .93

39341.839 .21

$7 \quad 39241.058 .87$

$4 \quad 40143.168 .35$

$4 \quad 41244.06 \quad 6.84$

$\begin{array}{lll}6 & 42745.49 & 5.8\end{array}$

53657.418 .94

545047.637 .57

45449.978 .29

940643.047 .98

639341.468 .96

$5 \quad 43436.8310 .45$

$3 \quad 43346.395 .39$

$4 \quad 45749.535 .17$

$6 \quad 40141.448 .15$

$6 \quad 39541.509 .02$

$5 \quad 44948.495 .71$

39441.198 .91

340141.568 .53

45449.435 .38

$43346.28 \quad 5.38$

548652.835 .66

45749.525 .17

$4 \quad 43346.235 .38$

64948.355 .14

253157.969 .55

45249.486 .51

$5 \quad 41644.40 \quad 8.85$

345148.795 .72

$244347.75 \quad 4.9$

53157.999 .48

45749.806 .75

644047.168 .95

347651.295 .63

64948.20501

353157.939 .55

$6 \quad 45749.836 .79$

$6 \quad 39942.628 .86$

$53157.36 \quad 6.7$

$4 \quad 52555.785 .26$

341643.838 .65

49554.248 .82

$6 \quad 49953.045 .73$

42444.558 .62

5
43146.718 .55

6
41643.748 .64

$\begin{array}{ll}3 & 49554.319 .08\end{array}$

452155.616 .7

342045.318 .85

340542.406 .45

$\begin{array}{lll}3 & 34538.25 & 5.57\end{array}$

$6 \quad 48851.865 .15$

Page 19/28 


\begin{tabular}{llllll} 
TaPG105TraesCS7D02G270500.1 256219076256220499 & 1215 & 3 & 42044.12 & 8.21 \\
TaPG106 TraesCS7D02G320900.1411009415411012798 & 1458 & 5 & 32033.59 & 8.66 \\
TaPG107TraesCS7D02G326800.1417356133417359468 & 1467 & 6 & 29430.79 & 6.81 \\
TaPG108TraesCS7D02G397900.1513311905513313583 & 1248 & 3 & 42044.12 & 8.04 \\
TaPG109TraesCS7D02G398100.1514074452514076294 & 1392 & 4 & 42045.48 & 8.91 \\
TaPG110TraesCS7D02G398900.1515219721515221824 & 1245 & 3 & 43946.63 & 8.32 \\
TaPG111TraesCS7D02G399000.1515235925515238032 & 1245 & 3 & 48551.98 & 6.35 \\
TaPG112 TraesCSU02G066000.1 51233915 51237421 & 1581 & 2 & 48751.75 & 5.07 \\
TaPG113 TraesCSU02G113500.1 97473524 & 97479283 & 1587 & 2 & 42044.23 & 6.26 \\
\hline
\end{tabular}

Start locus and End locus represent the start and end positions of the gene on the chromosome, respectively. CDS Length represents the length of the coding sequence of the corresponding gene, in bp. Exon: number of exons of the gene; aa: length of amino acid; MW: molecular mass; pI: isoelectric point.

Table 2. Ka/Ks values of tandem duplication TaPGs.

\begin{tabular}{ccccc}
\hline Name & Transcript ID & Ka & Ks & Ka/Ks \\
\hline TaPG19\&TaPG20 & TraesCS2B02G423200.1\&TraesCS2B02G423300.1 & 0.978963 & 1.05556 & 0.927433 \\
TaPG20\&TaPG21 & TraesCS2B02G423300.1\&TraesCS2B02G423400.1 & 0.0670902 & 0.274587 & 0.244332 \\
TaPG24\&TaPG25 & TraesCS2D02G402000.1\&TraesCS2D02G402100.1 & 0.960948 & 1.11393 & 0.862667 \\
TaPG35\&TaPG36 & TraesCS3B02G148500.1\&TraesCS3B02G148600.1 & 0.12591 & 0.337925 & 0.372598 \\
TaPG42\&TaPG43 & TraesCS3D02G130300.1\&TraesCS3D02G130400.1 & 0.130337 & 0.337546 & 0.386131 \\
TaPG93\&TaPG94 & TraesCS7A02G404900.1\&TraesCS7A02G405000.1 & 0.0106235 & 0.0757237 & 0.140293 \\
TaPG101\&TaPG102 & TraesCS7B02G304400.1\&TraesCS7B02G304500.1 & 0.00176716 & 0.0581157 & 0.0304077 \\
TaPG110\&TaPG111 & TraesCS7D02G398900.1\&TraesCS7D02G399000.1 & 0.00367189 & 0.0136337 & 0.269325 \\
\hline
\end{tabular}

Ka: non-synonymous mutation frequency; Ks: synonymous mutation frequency; Ka/Ks: ratio of non-synonymous mutation rate to synonymous mutation rate. This ratio can be used to determine if there is a selective pressure on this protein-coding gene.

\section{Additional Files}

Additional file 1: Table S1. Amino acid sequences of 113 wheat PGs.

Additional file 2: Figure S1. Position distribution of $113 \mathrm{TaPGs}$ on chromosomes.

Additional file 3: Table S2. Position information of wheat PG genes involved in segmental duplications.

Additional file 4: Figure S2. Ten motif sequences of wheat PGs.

Additional file 5: Table S3. Details of the GO annotation of the TaPGs.

Additional file 6: Figure S3. RT-PCR verification.

Additional file 7: Table S4. Gene annotations and interaction annotations of Arabidopsis proteins that with similar sequences to the important TaPGs. 


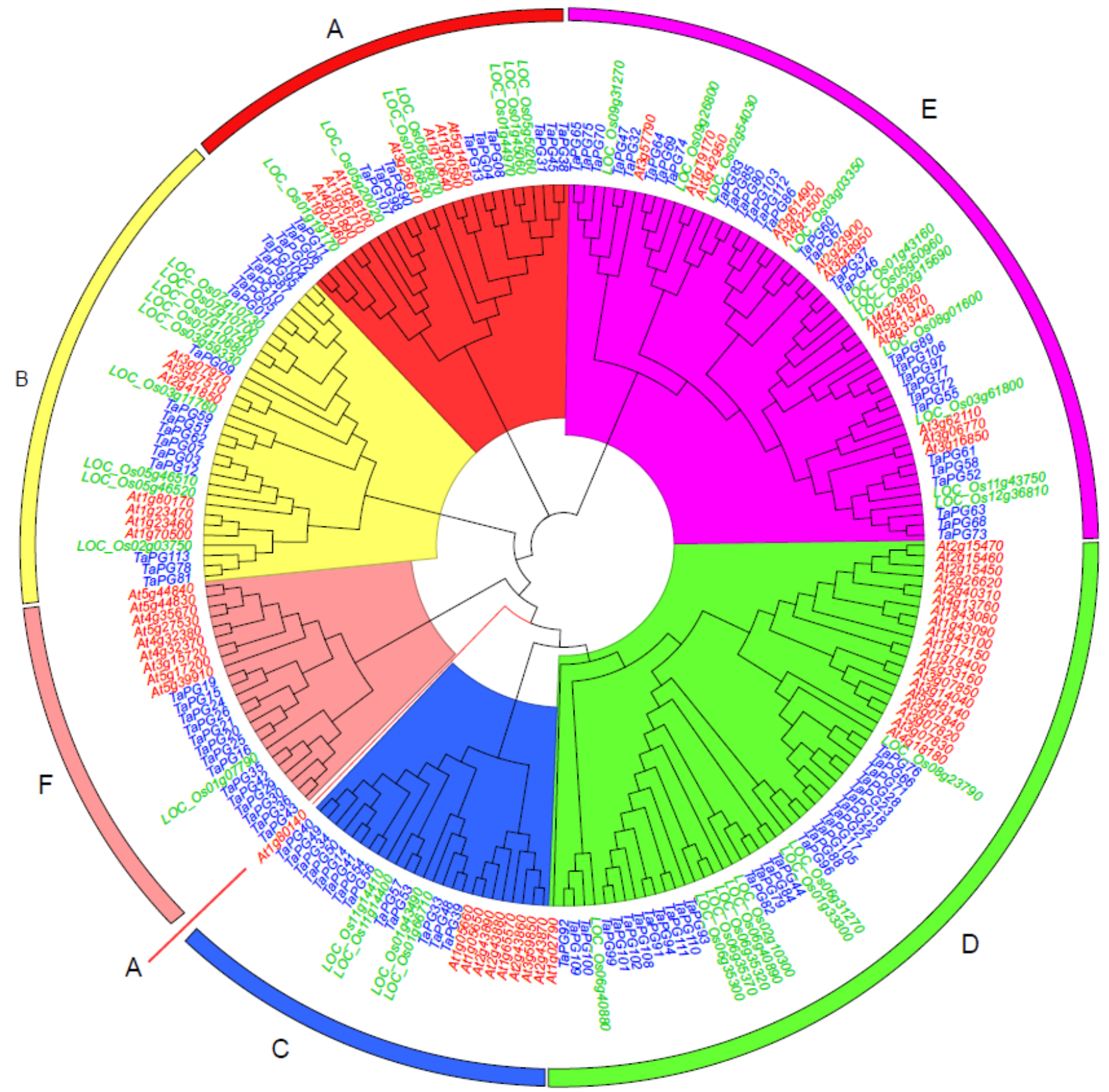

\section{Figure 1}

Phylogenetic tree of Arabidopsis thaliana, rice and wheat PGs. The shades of different colors on the tree are used to distinguish between different branches, and the letters A-F indicate the classification of the $P G$ gene family. 


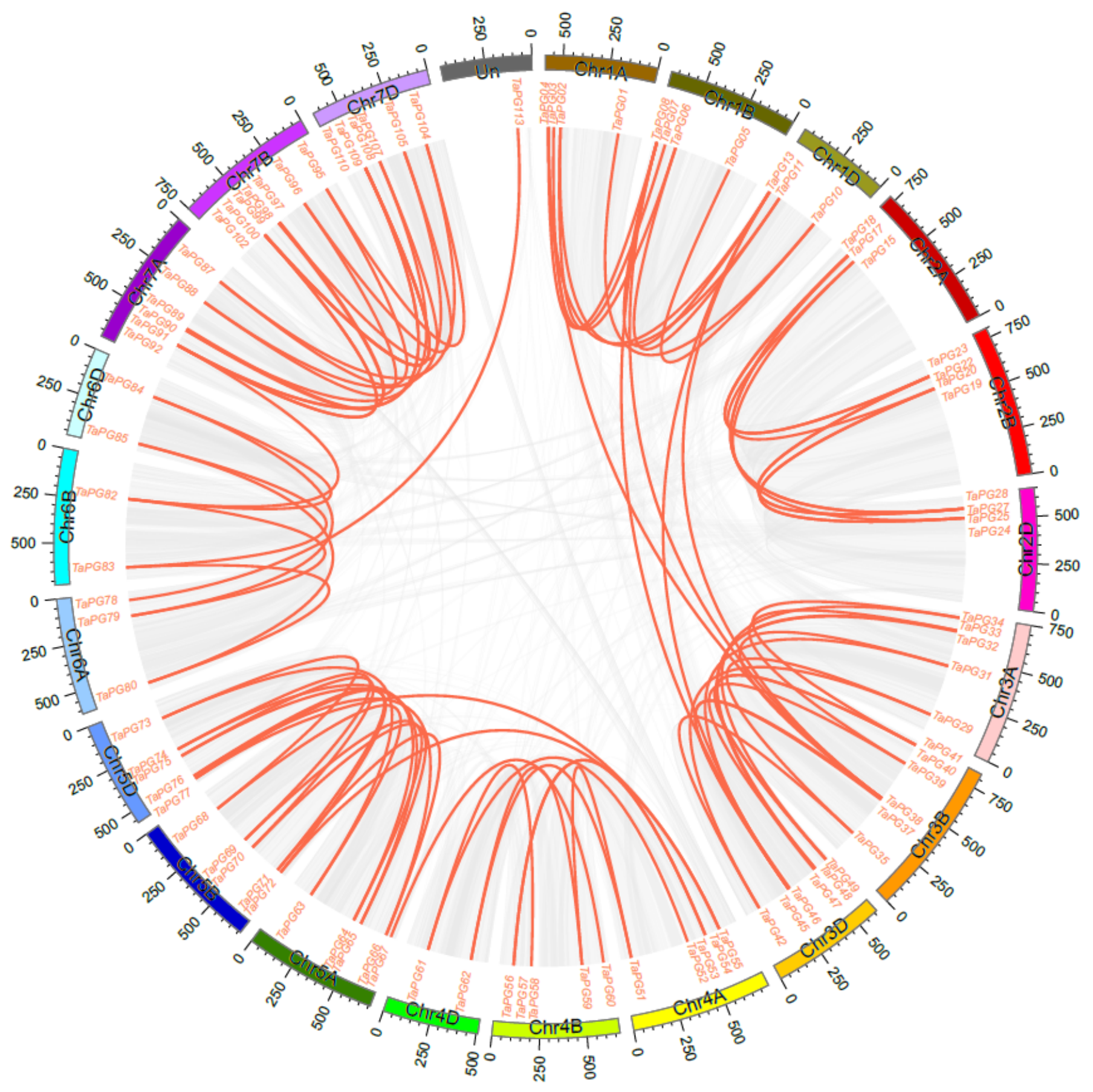

Figure 2

The synteny relationships of the wheat PGs. The gray lines in the middle represent the synteny of the whole wheat genome, and the orange lines represent the synteny between the PGs. 


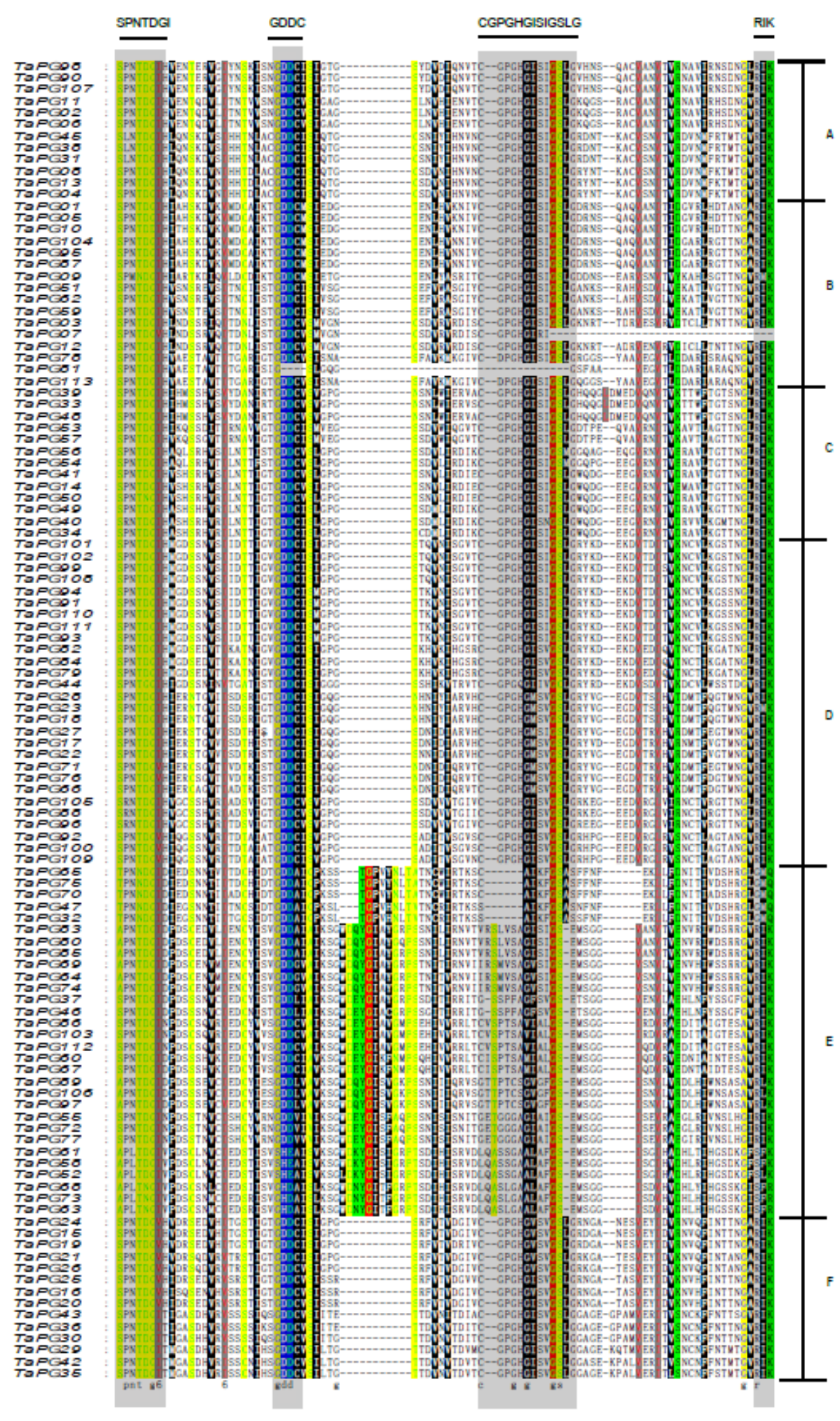

Figure 3

Amino acid sequences of four conserved domains of wheat PGs. The top notes are the conserved sequences of the four conserved domains in plant PG gene family, and the right side annotation is the different classification of PGs obtained from the phylogenetic tree. 

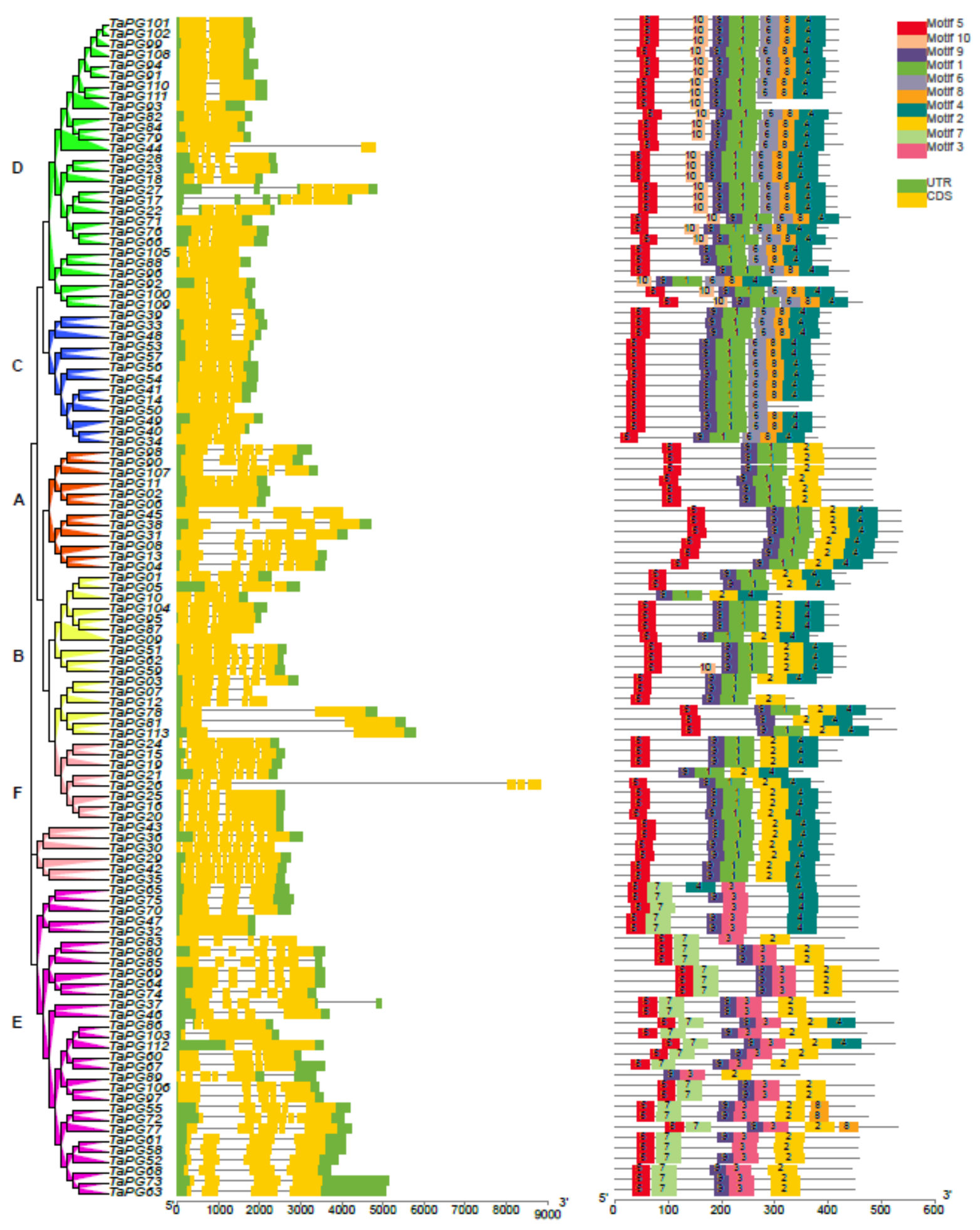

\section{Figure 4}

The gene structures of TaPGs and the conserved motifs of wheat PG proteins. The three parts from left to right are the phylogenetic tree of wheat PG gene family, the distribution of CDS and introns on TaPGs, the motifs distribution on the amino acid sequences of PGs. The scales at the bottom represents the genes length, and the numbers on the motifs represent the name of the motifs. 


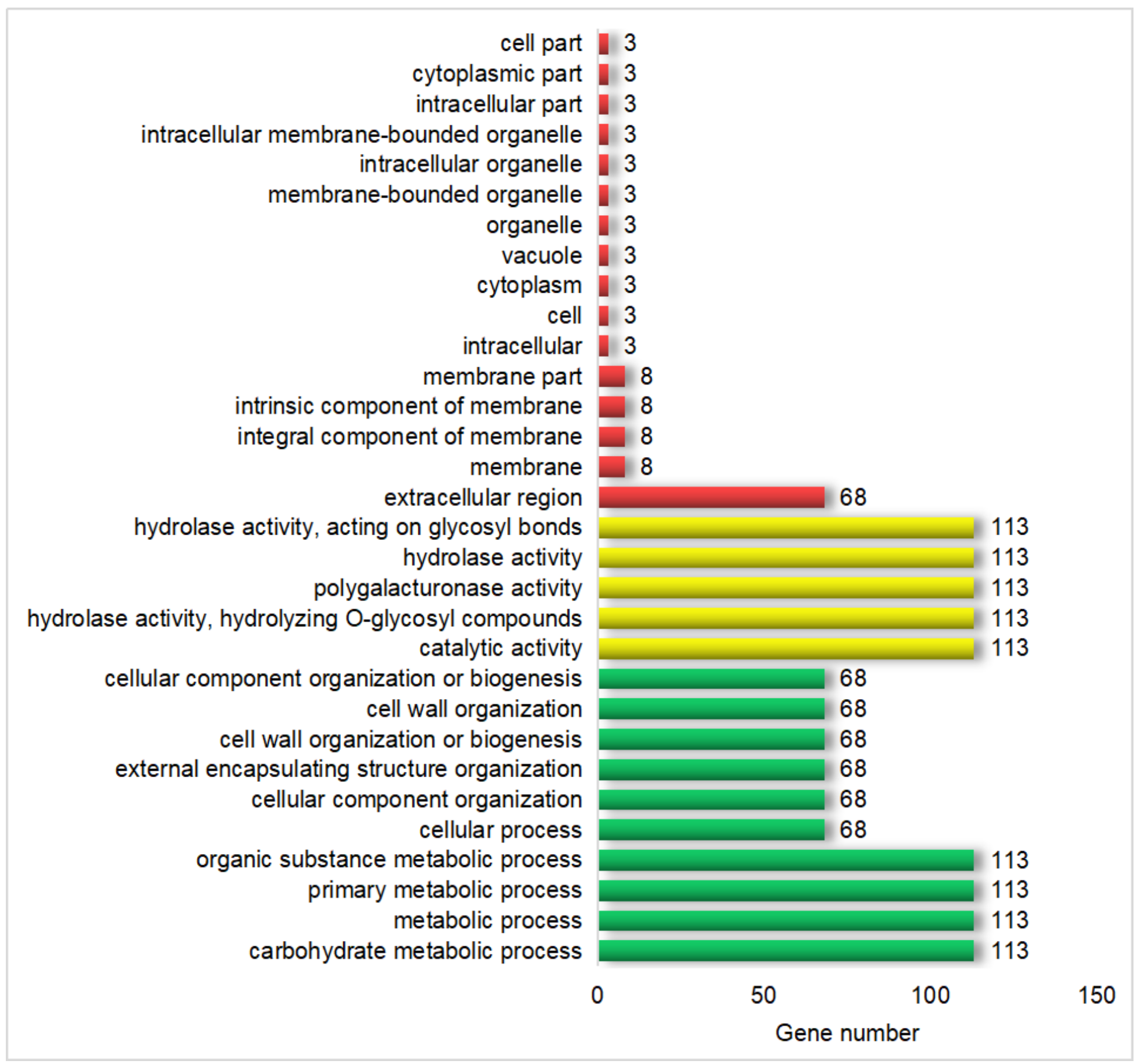

\section{Figure 5}

The GO annotations for TaPGs. The red, yellow and green bars represent the cellular components, molecular functions and biological processes of the TaPGs, respectively. The numbers at the end of the bars represent the number of wheat PG genes enriched to the corresponding annotation. 


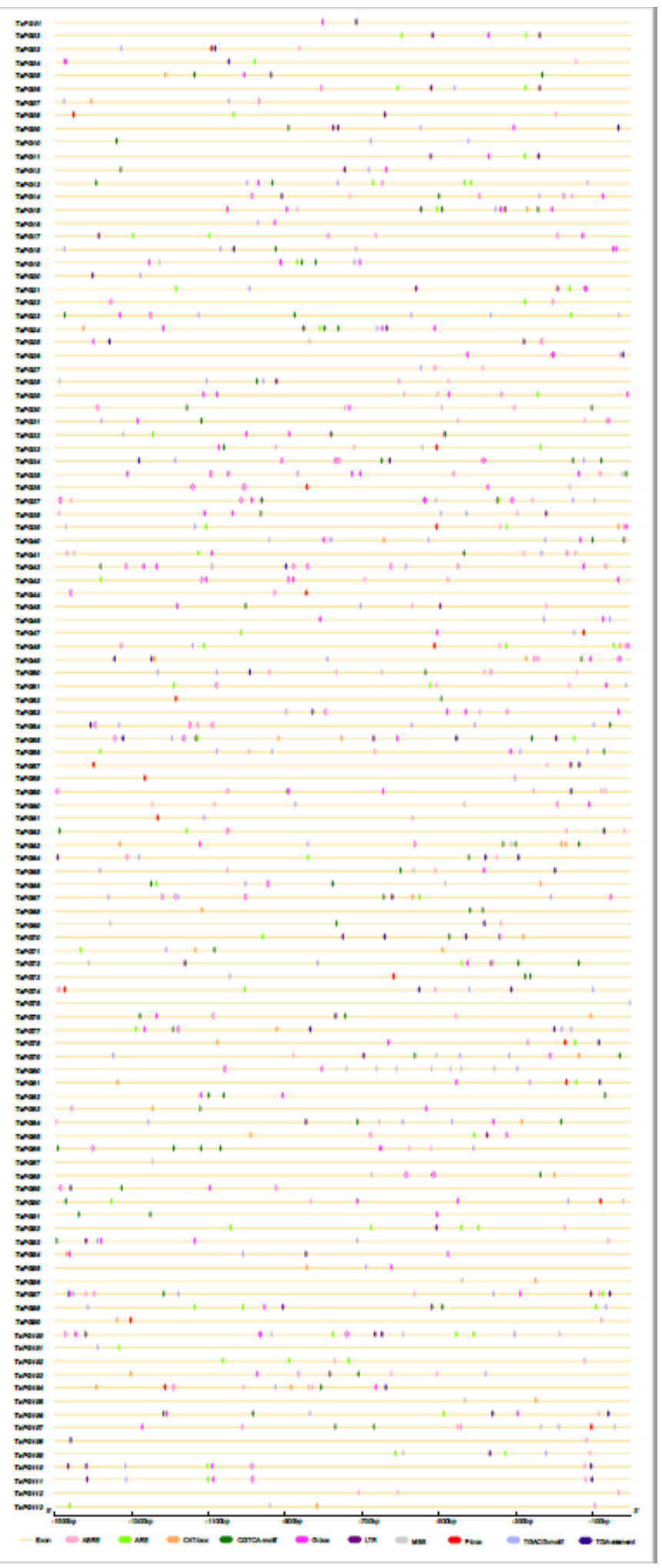

Figure 6

Distribution of cis-elements of TaPGs. The details of the cis-elements are represented by different color legends at the bottom. 


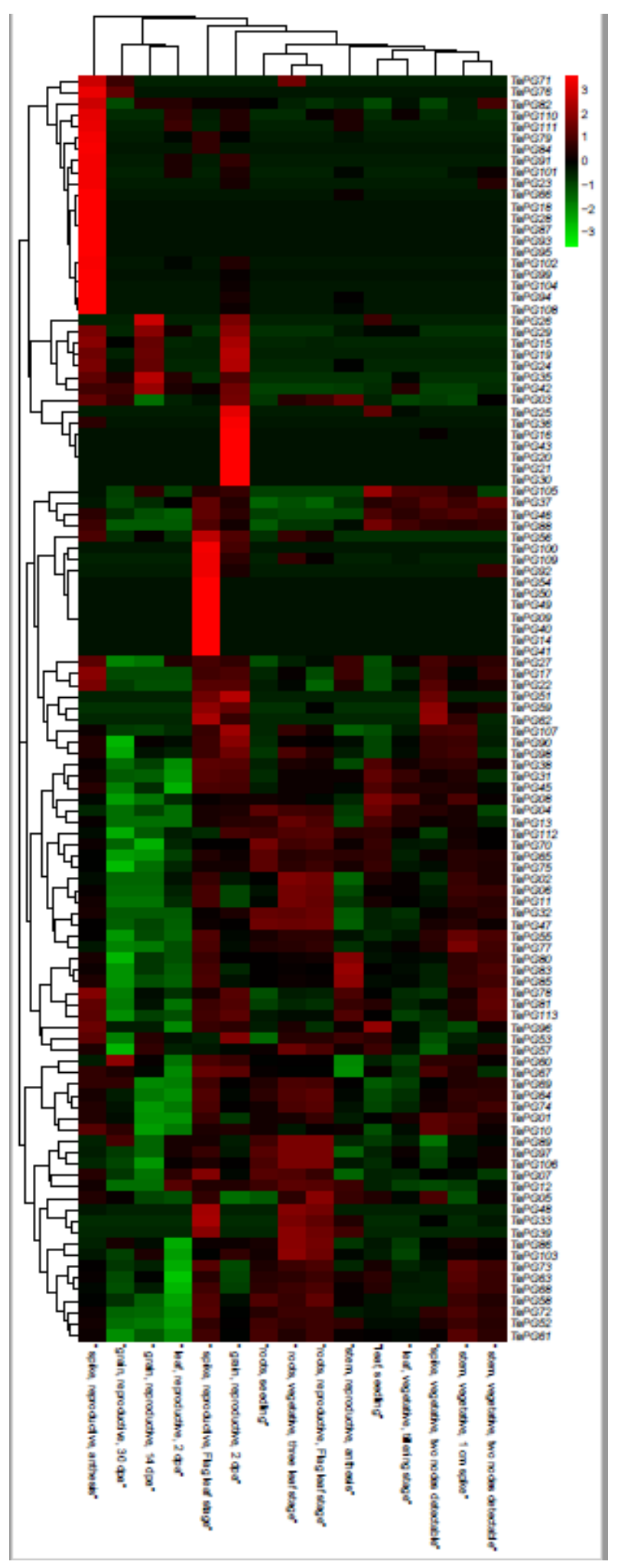

Figure 7

Heatmap of TaPGs in 15 different developmental stages and tissues. 


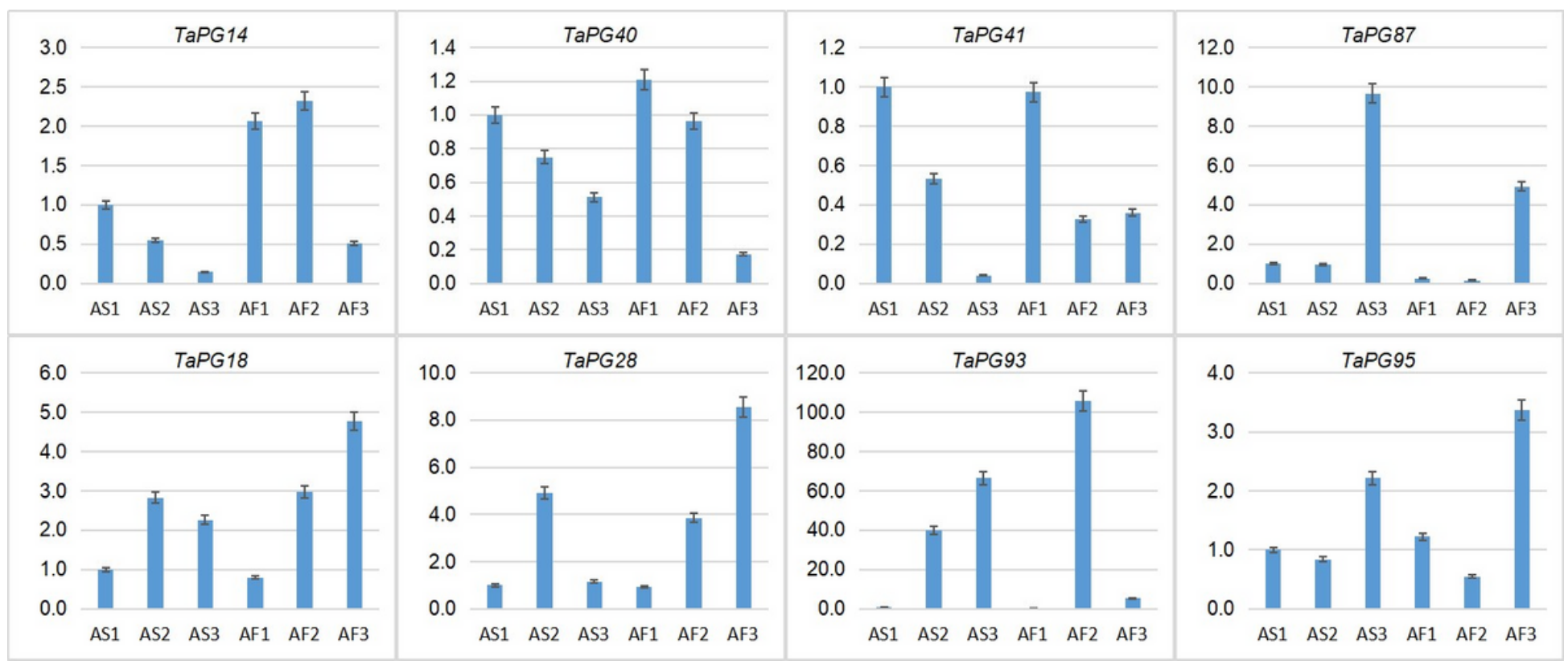

Figure 8

qRT-PCR results of 10 TaPGs.

\section{Supplementary Files}

This is a list of supplementary files associated with this preprint. Click to download.

- Additionalfile4FigureS2.png

- Additionalfile3TableS2.xlsx

- Additionalfile1TableS1.xIsx

- Additionalfile6FigureS3.png

- Additionalfile7Tables4.xlsx

- Additionalfile5Tables3.xls

- Additionalfile2Figures1.pdf 